\title{
Observing the spin Coulomb drag in spin-valve devices
}

\author{
G. Vignale* \\ Department of Physics and Astronomy, University of Missouri, Columbia, Missouri 65211, USA
}

(Received 24 August 2004; published 4 March 2005)

\begin{abstract}
The Coulomb interaction between electrons of opposite spin orientations in a metal or in a doped semiconductor results in a negative off-diagonal component of the electrical resistivity matrix - the so-called "spindrag resistivity." It is generally quite difficult to separate the spin-drag contribution from more conventional mechanisms of resistivity. In this paper I discuss two methods to accomplish this separation in a spin-valve device.
\end{abstract}

DOI: 10.1103/PhysRevB.71.125103

PACS number(s): 72.10.- d, 72.25.Dc

It is theoretically well established ${ }^{1-5}$ that the Coulomb interaction between electrons in a metal or in a doped semiconductor has a deeper effect on spin-polarized currents than on ordinary spin-unpolarized ones. The main reason for this is that the difference between the momenta of the up-spin and down-spin electrons is not conserved in a Coulomb scattering event: the transfer of momentum between electrons of opposite spin orientations therefore provides an intrinsic mechanism for the decay of a spin current, even in the absence of electron-impurity scattering. This effect has been called "spin Coulomb drag,"1 or just spin drag for brevity. Mathematically, the spin-drag effect is best described in terms of the so-called spin transresistivity $\rho_{\uparrow \downarrow}$, which is defined as follows: Let $j_{\uparrow}$ and $j_{\downarrow}$ be the electrical currents associated with up- and down-spin electrons (we consider here for simplicity only currents in the $x$ direction and neglect spin-orbit effects), and let $E_{\uparrow}, E_{\downarrow}$ be the electrochemical fields acting on the up and down spins, respectively. (The electrochemical field $E_{\sigma}$ is defined as the gradient of the electrochemical potential $\mu_{\sigma}$ divided by $e$. The electrochemical potential itself is the sum of the true electric potential, which determines the position of the bottom of the conduction band, and the chemical potential, which determines the level of occupation of the band.) Then, for small departures from equilibrium one has

$$
E_{\sigma}=\sum_{\sigma^{\prime}} \rho_{\sigma \sigma^{\prime}} j_{\sigma^{\prime}}
$$

where the resistivity matrix $\rho_{\sigma \sigma^{\prime}}$ has the form ${ }^{3}$

$$
\rho=\frac{m^{*}}{n e^{2} \tau}\left(\begin{array}{cc}
2+\gamma \tau & -\gamma \tau \\
-\gamma \tau & 2+\gamma \tau
\end{array}\right) .
$$

In the above equation $\gamma$ is the spin-drag coefficient, i.e., the intrinsic relaxation rate of the spin momentum $p_{\uparrow}-p_{\downarrow}, 1 / \tau$ is the ordinary momentum relaxation rate due to electron impurity interactions, $m^{*}$ and $e$ are the band mass and the absolute value of the electron charge, and $n$ is the total electronic density. Equation (2) is valid under the assumption that the spin-flip scattering rate is negligible in comparison to $\gamma$-a condition that should be well satisfied except at very low temperatures. ${ }^{3,4,6}$ We have also assumed, for simplicity, that the system is paramagnetic, i.e., $n_{\uparrow}=n_{\downarrow}=n / 2$, so that $\rho_{\uparrow \uparrow}=\rho_{\downarrow \downarrow}$. Looking at Eq. (2) we notice an important fact: $\boldsymbol{\rho}_{\uparrow \downarrow}$ is negative, because it takes a negative electrochemical field to prevent an up-spin current from flowing when a downspin current is present. On the other hand, the positivity of dissipation requires both eigenvalues of $\rho$ to be positive- $-\mathrm{a}$ condition that is obviously satisfied by Eq. (2) provided $\gamma$ is positive.

It is clear that an experimental determination of $\gamma$ would be of great interest since the value of this quantity is controlled by many-body correlations, which are intrinsic to the equilibrium state of the electron liquid. The main difficulty is that the spin transresistivity cancels out in the ordinary resistivity $\rho=\left(\rho_{\uparrow \uparrow}+\rho_{\uparrow \downarrow}\right) / 2$, so one has to devise an experiment that is somehow sensitive to the "spin resistivity" $\rho_{\text {spin }}$ $=\left(\rho_{\uparrow \uparrow}-\rho_{\uparrow \downarrow}\right) / 2=\rho(1+\gamma \tau)$. An obvious way to proceed, proposed in Ref. 1, is to measure the electrochemical potential difference in the down-spin component when an up-spinpolarized current is driven into the semiconductor via highly spin-polarized ferromagnetic electrodes (spin injectors). In the limit that the level of spin-polarization $p$ of the ferromagnetic electrodes is 100\% "up," and the spin diffusion length of the semiconductor is much larger than its geometrical length, the injected current is entirely in the up-spin component. Under these conditions, the electrochemical potential "drop" for down spins will be negative, if $\gamma$ is a finite positive quantity, and will vanish if $\gamma=0$. If, on the other hand, the polarization of the electrodes is less than $100 \%$ then the down-spin electrochemical potential drop may remain positive, foiling our attempts to detect and measure $\gamma$.

Thus, a very important question concerning this proposal is: how large should the polarization of the electrodes be so that one may observe a negative down-spin electrochemical potential difference (as opposed to the trivially positive electric potential difference)?

This paper is largely devoted to providing a sharp answer to this question. It will be shown [see Eqs. (9) and (10) below] that as $p$ increases from 0 to $100 \%$ there is a critical value of $p$, given by $p_{c} \cong 1 /(1+\gamma \tau)$, at which the electrochemical potential drop for down spins switches from positive to negative. Measuring $p_{c}$ amounts therefore to a measurement of $\gamma \tau$. The experiment could be carried out in a three-layer spin-valve structure, ${ }^{7-11}$ such as the one shown in Fig. 1. The two electrodes/spin injectors could be made out of a large-g-factor II-VI semiconductor, e.g., $\mathrm{Be}_{x} \mathrm{Mn}_{y} \mathrm{Zn}_{1-x-y} \mathrm{Se}$, where $g \sim 100,{ }^{12}$ which can be completely 


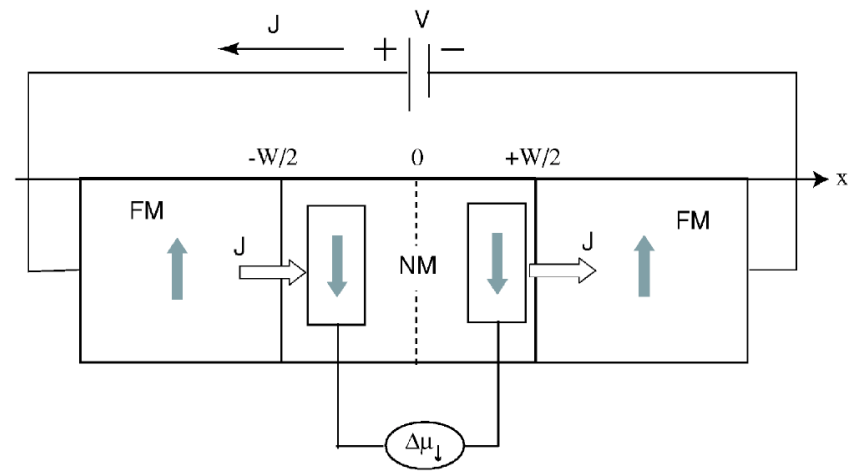

FIG. 1. Spin-valve device for the measurement of the spin drag effect. A predominantly up-spin current is injected in a nonmagnetic (NM) semiconductor via ferromagnetic (FM) electrodes between which a potential difference $V$ is applied. The voltage probes are polarized opposite to the injectors and therefore measure the down-spin electrochemical potential.

polarized by the application of a modest magnetic field. These electrodes are used to inject a spin-polarized current into a non-magnetic (NM) lightly doped semiconductor (e.g., GaAs) and the total resistance across the electrodes is measured. The main physical assumptions underlying the proposed measurement are as follows.

(1) The spin drag effect is important only in the nonmagnetic semiconductor (GaAs), where the density of carriers is low. This is because it is theoretically well established that the spin drag increases in magnitude as the density of the electrons decreases. ${ }^{1}$

(2) The magnetic field, which is needed to spin polarize the electrodes, has a negligible effect on the electronic states in the non-magnetic semiconductor, in which the $g$ factor is small.

(3) The spin-resolved conductivities of the electrodes $\sigma_{\uparrow \uparrow}^{f}$ and $\sigma_{\downarrow \downarrow}^{f}$ scale in proportion to the corresponding electron densities, i.e., $\sigma_{\uparrow \uparrow}^{f}=[(1+p) / 2] \sigma^{f}$ and $\sigma_{\downarrow \downarrow}^{f}=[(1-p) / 2] \sigma^{f}$, where $\sigma^{f}$ is the total conductivity of the homogeneous ferromagnet. (Of course $\sigma^{f}$ itself may slightly depend on $p$ : this question will be discussed below.)

The analysis is based on the equation for the electrochemical potentials derived in Ref. 13. In the onedimensional geometry of Fig. 1 this takes the form

$$
\frac{d^{2} \mu_{\sigma}(x)}{d x^{2}}=\sum_{\sigma^{\prime}} M_{\sigma \sigma^{\prime}} \mu_{\sigma^{\prime}}
$$

where the $2 \times 2$ matrix $M_{\sigma \sigma^{\prime}}$ is, for our purposes, completely specified by its right eigenvectors, namely

$$
\left(\begin{array}{l}
1 \\
1
\end{array}\right)
$$

(the charge mode) with eigenvalue 0 , and

$$
\left(\begin{array}{c}
1 \\
-1
\end{array}\right)
$$

(the spin mode) with eigenvalue $1 / L^{2}$, where $L$ is the spin diffusion length. The solution of Eq. (3) is straightforward. To make the best use of symmetry we assume that the semiconductor layer extends from $x=-W / 2$ to $x=W / 2$. The electrochemical potentials are then odd functions of $x\left[\mu_{\alpha}(x)\right.$ $\left.=-\mu_{\alpha}(-x)\right]$, and we can focus only on the region $x<0$. In this region we write

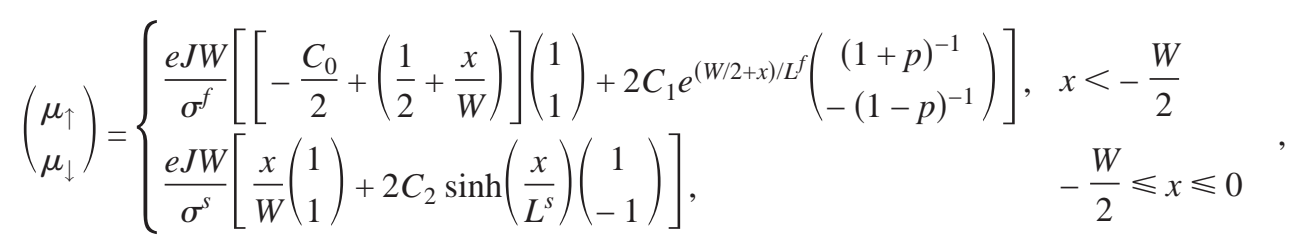

where $J$ is the charge current, $\sigma^{f}$ and $\sigma^{s}$ are the conductivities of the electrodes and of the semiconductor, and $L^{f}$ and $L^{s}$ the spin diffusion lengths in the electrodes and in the semiconductor, respectively. Notice that the continuity of the charge current, $J$, is already built in Eq. (6). The three constants $C_{0}, C_{1}$, and $C_{2}$ are determined from the continuity of the two electrochemical potentials and of the spin current $j_{\uparrow}(x)-j_{\downarrow}(x)$ at $x=-W / 2$. Their explicit forms are easily found to be

$$
\begin{gathered}
C_{1}=-\frac{p\left(1-p^{2}\right)}{2 \mathcal{D}} \sinh \left(\frac{W}{2 L^{s}}\right), \\
C_{2}=\frac{p \sigma^{s}}{2 \sigma^{f} \mathcal{D}},
\end{gathered}
$$

with

$$
\mathcal{D}=\frac{W\left(1-p^{2}\right)}{L^{f}} \sinh \left(\frac{W}{2 L^{s}}\right)+\frac{W \sigma^{s}}{L^{s} \sigma^{f}} \frac{1}{1+\gamma \tau} \cosh \left(\frac{W}{2 L^{s}}\right) .
$$

$$
C_{0}=\frac{\sigma^{f}}{\sigma^{s}}+\frac{2 p^{2}}{\mathcal{D}} \sinh \left(\frac{W}{2 L^{s}}\right)
$$

As mentioned above, the solution for $x>0$ is obtained by means of the symmetry relation $\mu_{\sigma}(x)=-\mu_{\sigma}(-x)$. 
The behavior of the solution (expressed in units of $\left.e J W / \sigma^{s}\right)$ is shown in Fig. 2. Basically, we observe an accumulation of down-spin electrons and a corresponding depletion of up-spin electrons (i.e., $\mu_{\downarrow}>\mu_{\uparrow}$ ) at the left interface, where up-spin electrons are injected. The opposite happens at the left interface, where up-spin electrons are extracted. These spin accumulations effectively create a diffusion barrier, which increases the resistance and reduces the efficiency of spin-current injection. Under the assumption $L^{s} \gg W$ the electrochemical fields, defined as the slopes of the elctrochemical potentials divided by $e$, are nearly exactly uniform in the non-magnetic region and their values are given by

$$
\begin{aligned}
& E_{\uparrow}(0)=\frac{J}{\sigma^{s}}+\frac{J W p}{L^{s} \sigma^{f} \mathcal{D}}, \\
& E_{\downarrow}(0)=\frac{J}{\sigma^{s}}-\frac{J W p}{L^{s} \sigma^{f} \mathcal{D}} .
\end{aligned}
$$

Notice that $E_{\downarrow}$ is always smaller than $E_{\uparrow}$ and would tend to zero for $p \rightarrow 100 \%$ in the absence of the spin drag effect. This is because as the polarization of the electrodes increases, the down-spin component of the current must decrease: in the absence of spin drag this would imply that a gradient in chemical potential of down-spin electrons must be present to balance the electric field, resulting in $E_{\downarrow} \approx 0$. The spin drag upsets this balance. It is now necessary to have a finite, negative $E_{\downarrow}$ in order to balance the momentum transfer from up- to down-spin electrons. The change in sign in $E_{j}$ is an unmistakable signature of the spin Coulomb drag and occurs when the spin polarization of the electrodes exceeds the critical value

$$
p_{c}=\frac{\sqrt{1+4 \alpha^{2} \sinh ^{2}\left(\frac{W}{2 L^{s}}\right)+2 \alpha \sinh \left(\frac{W}{L^{s}}\right) \frac{1}{1+\gamma \tau}}-1}{2 \alpha \sinh \left(\frac{W}{2 L^{s}}\right)}
$$

$$
\stackrel{W \ll L^{s}}{\simeq} \frac{1}{1+\gamma \tau},
$$

where $\alpha \equiv L^{s} \sigma^{f} / L^{f} \sigma^{s}$ (notice that $p_{c}>1$ for $\gamma=0$, that is, in the absence of spin drag. For $\gamma \tau=1$ with the parameters of Fig. 2 we have $p_{c} \approx 0.85$ ). Thus by measuring the value of $p$ at which $E_{\downarrow}(0)$ changes sign one can determine $\gamma \tau$.

The main drawback of such an experimental design (which is conceptually analogous to the design of the Coulomb drag measurement in bilayer systems ${ }^{14}$ ) is the need to establish separate electrical contacts for the up- and downspin electrons. This could be accomplished by the introduction of ferromagnetic voltage probes, polarized opposite to the current leads. Unfortunately, such "probes" are technically difficult to implant and complicate the analysis of the experiment, for they disturb the equilibrium distribution of the spin in the semiconductor. For this reason I now describe what should be a simpler method to determine the quantity $1+\gamma \tau$. The idea of the measurement is simply to compare the total resistance $R$ of the circuit at $p=0$ (i.e., for unpolarized electrodes) and $p=1$ (i.e., $100 \%$ spin-polarized electrodes).

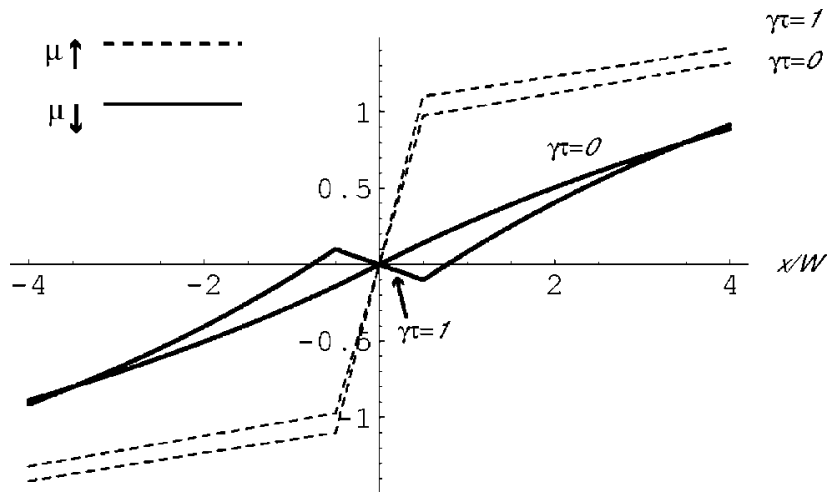

FIG. 2. The behavior of the electrochemical potentials $\mu_{\uparrow}$ (dashed lines) and $\mu_{\downarrow}$ (solid lines) calculated from Eq. (6) for $\gamma \tau$ $=0$ and $\gamma \tau=1$ and expressed in units of $e J W / \sigma^{s}$ in the parallelelectrodes configuration. The semiconductor is in the region $-1 / 2$ $\leqslant x / W \leqslant 1 / 2$. The other parameters are $p=90 \%, \sigma^{f} / \sigma^{s}=10, L^{s} / W$ $=2$, and $L^{f} / W=4$. Noticethe negative slope of $\mu_{\downarrow}(x)$ at $x=0$ when $\gamma \tau=1$ : such a negative slope is an unmistakable signature of the spin drag.

No spin-polarized voltage probes are required. We assume that the homogeneous resistance of the electrodes and the external wires (denoted by $R_{c}$ for brevity) is small compared to the resistance of the non-magnetic semiconductor. The polarization dependence of $R_{c}$ presumably amounts to an even smaller correction. At $p=0$ the total resistance is thus essentially equal to the ordinary resistance of the semiconductor: the spin-drag effect is invisible here. At $p=1$, on the other hand, the resistance depends very much on whether there is spin drag or not. If the spin drag were absent, then the resistance would be twice the ordinary resistance of the semiconductor, because only one of the two spin channels is open to conduction. In the presence of spin drag the flow of the up-spin current is hindered by collisions with down-spin electrons, which are stationary on the average: as a result, the resistance of the conductor becomes more than twice the ordinary resistance-in fact we will show that it is $2+\gamma \tau$ times the ordinary resistance. Thus, by taking the difference $R(p=1)-R(p=0)$ and dividing it by $R(p=0)$ we arrive at an experimental determination of $\gamma \tau$. It should be noted that in taking the difference $R(p=1)-R(p=0)$ the resistance $R_{c}$ of the wires and the electrodes largely cancels out, except for its polarization-dependent component, which we feel justified in neglecting. Furthermore, this determination does not depend on the value of the spin diffusion length in the semiconductor, $L^{S}$, provided the latter is much larger than the length of the semiconductor itself - a condition that should not be too difficult to satisfy in practice. Likewise, the value of the spin-diffusion length in the electrodes, $L^{f}$, is essentially irrelevant as long as the potential drop is measured between points that are much farther than a distance $L^{f}$ from the FM-NM interfaces.

Far from the FM-NM interfaces (more precisely, at a distance much larger than $L^{f}$ ) the electrochemical potentials of the two spin orientations tend to common values $\mu_{-}$ $=-e J W\left(C_{0}-1\right) / 2 \sigma^{f}+e J x / \sigma^{f}$ for $x \rightarrow-\infty$ and $\mu_{+}=+e J W\left(C_{0}\right.$ $-1) / 2 \sigma^{f}+e J x / \sigma^{f}$ for $x \rightarrow+\infty$. The difference between these two asymptotic behaviors is $e$ times the voltage drop due to 
the presence of the semiconductor layer. Hence, the resistance of our device (per unit cross-sectional area) is given by

$$
R_{\text {parallel }}(p)=R_{c}(p)+R^{s}+\frac{2 W p^{2}}{\mathcal{D} \sigma^{f}} \sinh \left(\frac{W}{2 L^{s}}\right),
$$

where $R_{c}(p)$ is the combined resistance of the electrodes and the external wires, and $R^{s}=W / \sigma^{s}$ is the ordinary resistance of the semiconductor. The last term on the right hand side of this equation is due to the spin accumulations at the interfaces between the electrodes and the semiconductor. Figure 3 shows the behavior of the key quantity $R_{\text {parallel }}(p)-R_{c}(p)$ as a function of $p$. It increases from $R_{s}$ at $p=0$ to $R^{s}(2+\gamma \tau)$ at $p=1$. Interestingly most of the change occurs in the region of $p$ close to 1 . This can be exploited to reduce the undesired effect of the $p$ dependence of $R_{c}$. Namely, rather than considering the change in resistance from $p=0$ to $p=1$, it may be sufficient to consider the change from say $p=0.5$ to $p=1.0$ with correspondingly less variation in $R_{c}$. Notice that heoretical calculations of $\gamma$ as a function of temperature and electronic density can be found in Refs. 1-4. The temperature dependence of $\gamma$ is particularly interesting as it exhibits a characteristic broad maximum at about the degeneracy temperature of the carriers in the semiconductor.

For completeness, let us now see what happens in the antiparallel-electrodes configuration. In this case, the electro-

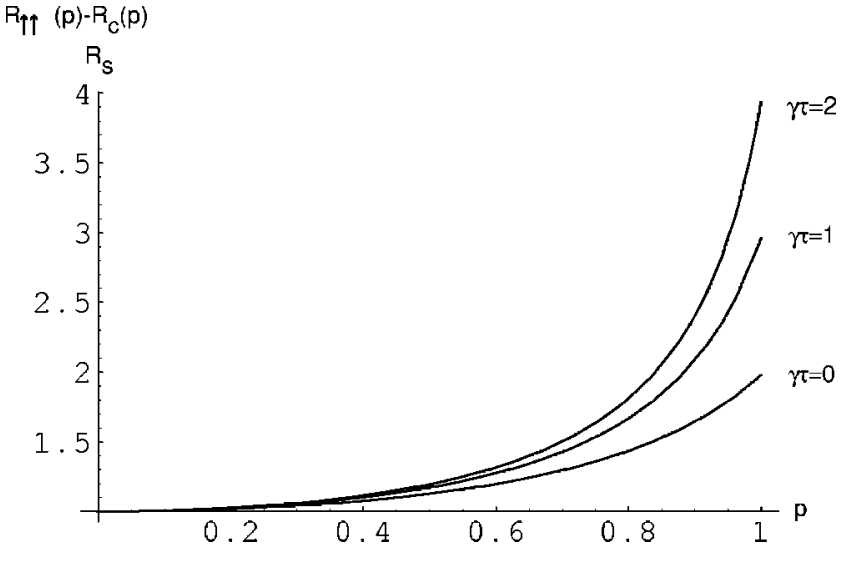

FIG. 3. Behavior of the parallel-electrodes magnetoresistance $R_{\text {parallel }}(p)-R_{c}(p)$ (in units of the ordinary resistance of the semiconductor, $R_{s}$ ) as a function of $p$ for $\gamma \tau=0,1$, and 2 . Notice the sharp enhancement caused by the spin-drag resistivity for values of $p$ close to $100 \%$. As explained in the text, this can be used to determine $\gamma \tau$.

chemical potentials obey the symmetry relation $\mu_{\sigma}(x)=$ $-\mu_{-\sigma}(-x)$ and it is easy to see that the new solution is now obtained from the parallel case solution simply by interchanging the quantities $\sinh \left(W / 2 L^{s}\right)$ and $\cosh \left(W / 2 L^{s}\right)$. More precisely, the solution for $x<0$ takes the form

$$
\left(\begin{array}{c}
\mu_{\uparrow} \\
\mu_{\downarrow}
\end{array}\right)=\left\{\begin{array}{cc}
\frac{e J W}{\sigma^{f}}\left\{\left[-\frac{C_{0}^{\prime}}{2}+\left(\frac{1}{2}+\frac{x}{W}\right)\right]\left(\begin{array}{l}
1 \\
1
\end{array}\right)+2 C_{1}^{\prime} e^{(W / 2+x) / L^{f}}\left[\begin{array}{c}
(1+p)^{-1} \\
-(1-p)^{-1}
\end{array}\right]\right\}, & x<-\frac{W}{2} \\
\frac{e J W}{\sigma^{s}}\left\{\frac{x}{W}\left(\begin{array}{l}
1 \\
1
\end{array}\right)-2 C_{2}^{\prime} \cosh \left(\frac{x}{L^{s}}\right)\left(\begin{array}{c}
1 \\
-1
\end{array}\right)\right\}, & -\frac{W}{2} \leqslant x \leqslant 0
\end{array},\right.
$$

where the constants $C_{0}^{\prime}-C_{2}^{\prime}$ are given by

$$
\begin{gathered}
C_{0}^{\prime}=\frac{\sigma^{f}}{\sigma^{s}}+\frac{2 p^{2}}{\mathcal{D}^{\prime}} \cosh \left(\frac{W}{2 L^{s}}\right), \\
C_{1}^{\prime}=-\frac{p\left(1-p^{2}\right)}{2 \mathcal{D}^{\prime}} \cosh \left(\frac{W}{2 L^{s}}\right), \\
C_{2}^{\prime}=\frac{p \sigma^{s}}{2 \sigma^{f} \mathcal{D}^{\prime}},
\end{gathered}
$$

and

$$
\mathcal{D}^{\prime}=\frac{W\left(1-p^{2}\right)}{L^{f}} \cosh \left(\frac{W}{2 L^{s}}\right)+\frac{W \sigma^{s}}{L^{s} \sigma^{f}} \frac{1}{1+\gamma \tau} \sinh \left(\frac{W}{2 L^{s}}\right) .
$$

The solution for $x>0$ is obtained by means of the symmetry relation $\mu_{\sigma}(x)=-\mu_{-\sigma}(-x)$. A representative plot of $\mu_{\uparrow}$ and $\mu_{\downarrow}$ is shown in Fig. 4.
We can calculate the resistance of the antiparallelelectrodes configuration in precisely the same way as in the parallel-electrodes case. The result is

$$
R_{\text {antiparallel }}=R_{c}(p)+R^{s}+\frac{2 W p^{2}}{\mathcal{D}^{\prime} \sigma^{f}} \sinh \left(\frac{W}{2 L^{s}}\right),
$$

and the quantity $R_{\text {antiparallel }}(p)-R_{c}(p)$ is plotted versus $p$ in Fig. 5. The resistance of this configuration is of course much larger than that of the parallel configuration [this is the well known giant magnetoresistance (GMR) effect] and it is easy to see that in the limit $p \rightarrow 1$ it tends to to $R^{s}(1+\gamma \tau)$ $\times\left(2 L^{s} / W\right)^{2}$. Notice that the Coulomb enhancement in this configuration is very sharply confined to the region of $p$ $\sim 1$. The results of the above calculation can be used to determine $L^{s} / W$, once $\gamma \tau$ has been determined from the measurement of the resistance in the parallel-electrodes configuration.

In summary I have theoretically analyzed in this paper two methods to measure the spin drag coefficient of a non- 


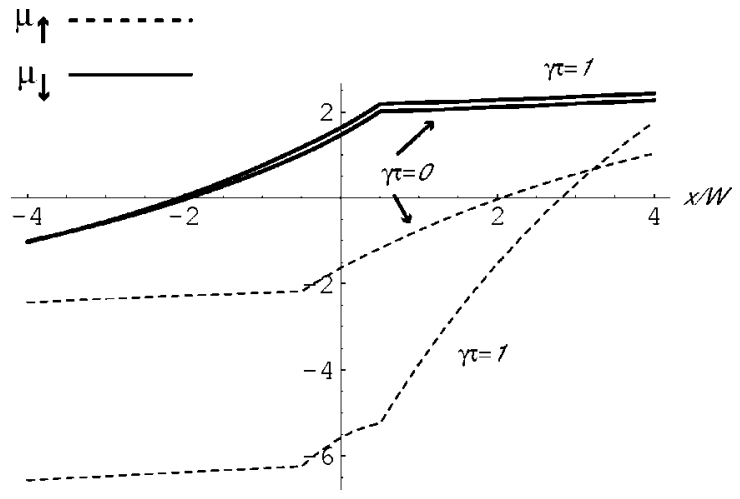

FIG. 4. The behavior of the electrochemical potentials $\mu_{\uparrow}$ (dashed lines) and $\mu_{\downarrow}$ (solid lines) calculated from Eq. (12) for $\gamma \tau$ $=0$ and $\gamma \tau=1$, expressed in units of $e J W / \sigma^{s}$ in the antiparallelelectrodes configuration. The parameters are the same as in the caption of Fig. 2, namely $p=90 \%, \sigma^{f} / \sigma^{s}=10, L^{s} / W=2$, and $L^{f} / W$ $=4$.

magnetic semiconductor sandwiched between highly spinpolarized ferromagnetic electrodes. The first method builds upon the gedanken experiment proposed in Ref. 1 showing that an unambiguous qualitative signature of the spin drag effect occurs when the spin polarization of the ferromagnetic electrodes exceed the critical value $p_{c} \simeq 1 /(1+\gamma \tau)$. In the second more quantitative method one simply measures the extra resistance introduced by the relative motion of the upspin and down-spin electrons in the semiconductor region of a basic spin-valve device. It is hoped that these discussions

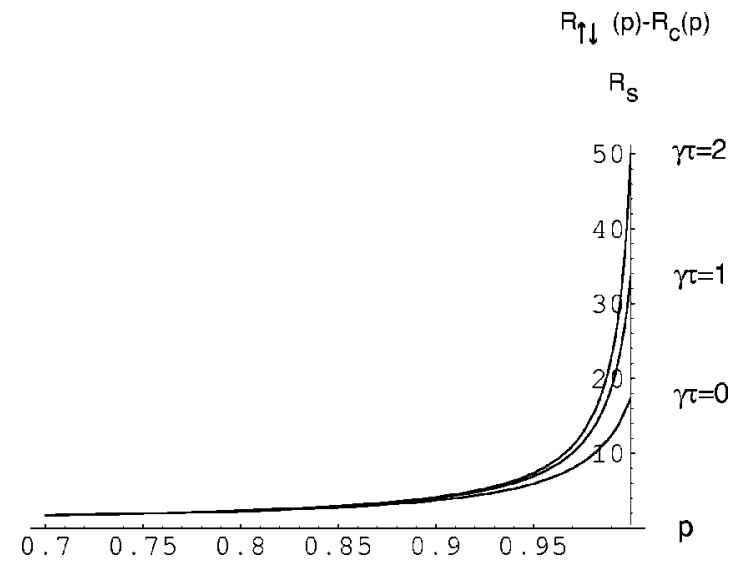

FIG. 5. Behavior of the antiparallel-electrodes magnetoresistance $R_{\text {parallel }}(p)-R_{c}(p)$ (in units of the ordinary resistance of the semiconductor, $R_{s}$ ) as a function of $p$ for $\gamma \tau=0,1$, and 2. The enhancement caused by the spin-drag resistivity for values of $p$ close to $100 \%$ is now amplified by a factor $\left(2 L^{s} / W\right)^{2}(=16$ in the present case). As explained in the text, this can be used to mesaure $L^{s} / W$ once $\gamma \tau$ is known.

will encourage further experimental work aimed at the observation of the spin Coulomb drag.

The author gratefully acknowledges the hospitality of the Center for Nanoscience of the University of Science and Technology of China, Hefei, China, where much of this work was completed, and support from NSF Grant No. DMR0313681. Many thanks also to Irene D'Amico for many useful comments on the manuscript.
*Email address: vignaleg@missouri.edu

${ }^{1}$ I. D'Amico and G. Vignale, Phys. Rev. B 62, 4853 (2000).

${ }^{2}$ I. D'Amico and G. Vignale, Europhys. Lett. 55, 566 (2001).

${ }^{3}$ I. D'Amico and G. Vignale, Phys. Rev. B 65, 085109 (2002).

${ }^{4}$ I. D'Amico and G. Vignale, Phys. Rev. B 68, 045307 (2003).

${ }^{5}$ K. Flensberg, T. S. Jensen, and N. A. Mortensen, Phys. Rev. B 64, 245308 (2001).

${ }^{6}$ The temperature dependence of the spin-drag resistivity is shown in Figs. 1 and 2 of Ref. 3 and in Fig. 1 of Ref. 4. A crude estimate of the spin-flip contribution to the off-diagonal resistivity gives $10^{-8} \Omega \mathrm{cm}$ in three-dimensional GaAs with carrier density $\sim 10^{17} \mathrm{~cm}^{-3}$ and spin relaxation time $\sim 50 \mathrm{~ns}$. This is five orders of magnitude smaller than the spin-drag resistivity in the same system.

${ }^{7}$ M. N. Baibich, J. M. Broto, A. Fert, F. Nguyen Van Dau, F. Petroff, P. Eitenne, G. Creuzet, A. Friederich, and J. Chazelas, Phys. Rev. Lett. 61, 2472 (1988).

${ }^{8}$ T. Valet and A. Fert, Phys. Rev. B 48, 7099 (1993).
${ }^{9}$ G. Schmidt, D. Ferrand, L. W. Molenkamp, A. T. Filip, and B. J. van Wees, Phys. Rev. B 62, R4790 (2000).

${ }^{10}$ G. Schmidt, G. Richter, P. Grabs, C. Gould, D. Ferrand, and L. W. Molenkamp, Phys. Rev. Lett. 87, 227203 (2001).

${ }^{11}$ For a review of the current situation in semiconductor spintronics see Semiconductor Spintronic and Quantum Computation, edited by D. D. Awschalom, N. Samarth, and D. Loss (SpringerVerlag, Berlin, 2002).

${ }^{12}$ R. Fielderling, M. Keim, G. Reuscher, W. Ossau, G. Schmidt, A. Waag, and L. W. Molenkamp, Nature (London) 402, 787 (1999).

${ }^{13}$ G. Vignale and I. D'Amico, Solid State Commun. 127, 829 (2003).

${ }^{14}$ T. J. Gramila, J. P. Eisenstein, A. H. MacDonald, L. N. Pfeiffer, and K. W. West, Phys. Rev. Lett. 66, 1216 (1991). For a review of the theoretical and experimental situation on Coulomb drag see A. G. Rojo, J. Phys.: Condens. Matter 11, R31 (1999). 